\title{
The Influence of Electrodes and Conditioning on Space Charge Accumulation in XLPE
}

\author{
Fleming, R. J.; Henriksen, Mogens; Holbøll, Joachim
}

Published in:

IEEE Transactions on Dielectrics and Electrical Insulation

Link to article, DOI:

10.1109/94.868078

Publication date:

2000

Document Version

Publisher's PDF, also known as Version of record

Link back to DTU Orbit

Citation (APA):

Fleming, R. J., Henriksen, M., \& Holbøll, J. (2000). The Influence of Electrodes and Conditioning on Space Charge Accumulation in XLPE. IEEE Transactions on Dielectrics and Electrical Insulation, 7(4), 561-571. https://doi.org/10.1109/94.868078

\section{General rights}

Copyright and moral rights for the publications made accessible in the public portal are retained by the authors and/or other copyright owners and it is a condition of accessing publications that users recognise and abide by the legal requirements associated with these rights.

- Users may download and print one copy of any publication from the public portal for the purpose of private study or research.

- You may not further distribute the material or use it for any profit-making activity or commercial gain

- You may freely distribute the URL identifying the publication in the public portal 


\title{
The Influence of Electrodes and Conditioning on Space Charge Accumulation in XLPE
}

\author{
R. J. Fleming, ${ }^{1}$ M. Henriksen and J. T. Holbøll \\ Department of Electric Power Engineering \\ Technical University of Denmark \\ Lyngby, Denmark
}

\begin{abstract}
The accumulation of space charge in planar crosslinked polyethylene (XLPE) samples under dc electric fields at room temperature was investigated using the pulsed electroacoustic (PEA) method. Three different organic semiconductor (semicon) materials containing carbon black at concentrations $\sim 30 \%$ wt, and vacuum-evaporated gold, were used as electrodes. Three different conditioning procedures were investigated. Unconditioned samples developed heterocharge with density increasing from zero at the electrodes, to a maximum at roughly onethird of the thickness, and then falling to zero around the center. They also developed homocharge close to the electrodes. The choice of electrode material had little effect on the heterocharge profile in unconditioned samples. Conditioning by holding at $80^{\circ} \mathrm{C}$ for four days, at rotary pump pressure or at atmospheric pressure, suppressed the accumulation of heterocharge. Homocharge accumulation close to the electrodes in samples with semicon electrodes was affected little by this conditioning, but was reduced considerably in samples with gold electrodes. Conditioning by holding at room temperature for seven days at rotary pump pressure had little effect. The heterocharge originates in inhomogeneous polarization due to a spatially inhomogeneous distribution of permanent dipole molecules, probably acetophenone. The dipole concentration decreases in going from the electrodes towards the center of the samples, as a result of diffusion of the molecules in the opposite direction. The homocharge originates in electronic charge injection/extraction across the electrode/XLPE interfaces.
\end{abstract}

\section{INTRODUCTION}

$S^{\mathrm{p}}$ PACE charge accumulation in crosslinked polyethylene (XLPE) has been studied extensively over the last ten years, following the development of reliable non-destructive methods of measuring the charge density, polarity and spatial profile over a wide range of sample thickness [1]. The main driving force behind this work has been the need to eliminate, or greatly reduce, the accumulation of space charge in XLPE under dc stress. Then its superior mechanical, chemical and other electrical properties could be exploited by using it as the insulant in $\mathrm{dc}$ power transmission cables.

The composition of the inner and outer semicon shields in modern ac cables, and the temperature/pressure conditions under which the various manufacturing procedures are carried out, would be expected to influence significantly the space charge accumulation under dc stress. Yet, only a few systematic investigations of these factors on space charge accumulation or other electrical properties of $\mathrm{XLPE}$, have been reported [2-7]. The authors of [2] concluded that the carbon charged polyethylene (PE) electrodes attached to their planar samples must be partially blocking in order to generate the reversal of the discharge (or resorption) currents reported by several authors in a range of PE. Also, an influence of the semicon electrodes on the conductivity of planar XLPE samples was inferred from the observation that the slopes of log-log plots of steady-state current density vs. applied electric field were dependent on whether the electrodes had been vacuum degassed [3]. Wang et al. [4] monitored space charge accumulation in lengths of freshly cured cylindrical power cable incorporating XLPE insulation, and found that previously accumulated space charge disappeared when the sample was held in short-circuit at temperatures in the range 25 to $80^{\circ} \mathrm{C}$ for $2 \mathrm{~h}$ in an air oven. However, the charge reappeared when the voltage was re-applied. On the other hand, holding the samples in short-circuit at $80^{\circ} \mathrm{C}$ and at pressures $\sim 1.3 \mathrm{~Pa}$ for 10 days also removed any pre-existing space charge, and prevented its reappearance for at least $2 \mathrm{~h}$ when the voltage was re-applied at room temperature. This result was attributed to removal of volatile impurities and modification of the crystalline/amorphous interfaces as a result of heating and pumping. Lim et al. [5], also working on commercial power cable samples with XLPE insulation, reported that pre-existing charge was removed when the sample was conditioned in short-circuit for $2 \mathrm{~h}$ at $82^{\circ} \mathrm{C}$ and atmospheric pressure, but, when voltage was reapplied, charge accumulated at a much faster rate than that observed before conditioning. Following conditioning in short-circuit for 10 days at $82^{\circ} \mathrm{C}$ and $0.1 \mathrm{~Pa}$, no space charge was detected until voltage had been applied for $\sim 40 \mathrm{~h}$, and its density increased very slowly. Interestingly, if the sample was then conditioned for $2 \mathrm{~h}$ at $82^{\circ} \mathrm{C}$ and atmospheric 

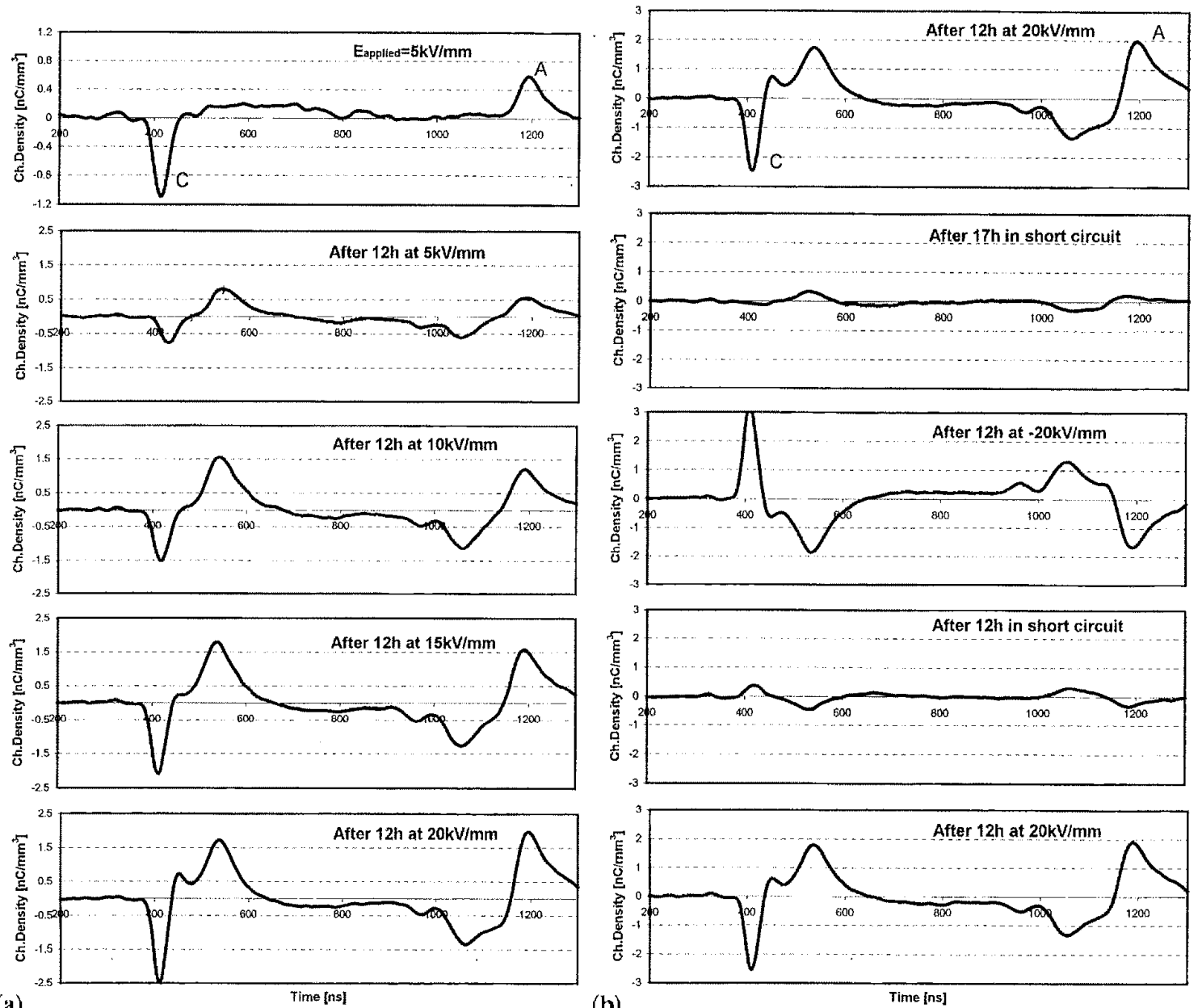

Figure 1. (a) Space charge profiles for an unconditioned sample with type A semicon electrodes. The top profile was obtained $\sim 5$ min after the field had been applied, and shows the charge induced on the cathode $(\mathrm{C})$ and anode $(\mathrm{A})$ by the applied field. The cathode was grounded and connected to the piezoelectric detector: The other profiles were obtained with the sample short-circuited, after the field had been applied as specified, and show the space charge in the sample bulk and the charge induced on the electrodes by the bulk charge. The time (horizontal) axis may be converted to distance from the cathode by multiplying by the speed of sound in XLPE $(\approx 2 \mathrm{~km} / \mathrm{s})$. No corrections have been applied for attenuation or dispersion of the pressure pulses in transit through the sample. (b) All profiles were obtained with the sample short-circuited, and show the effects of short-circuiting the sample for several hours, and reversing the applied field. The left-hand-side electrode was always grounded.

pressure, the charge re-appeared at much the same rate as after conditioning at $82^{\circ} \mathrm{C}$ and atmospheric pressure. Sanden [6] used a twinscrew extruder to produce tape from cable quality XLPE granules. The tapes were then pressure molded into cup-shaped samples, the planar bases being $1 \mathrm{~mm}$ thick with Rogowski-profile edges. The electrodes were made from semiconducting cable screen material and contained the same crosslinking agent and antioxidant as the XLPE. They were crosslinked to the XLPE during the pressure molding stage. Some of the samples were conditioned in an air-ventilated oven at $90^{\circ} \mathrm{C}$ for $72 \mathrm{~h}$. Space charge profiles for unconditioned and conditioned samples, subjected to dc fields in the range 20 to $70 \mathrm{kV} / \mathrm{mm}$ at $40^{\circ} \mathrm{C}$ for various times, were compared. The main conclusions were that (a) heterocharge formation originating in bulk processes was dominant in unconditioned samples, (b) homocharge injection from the electrodes was dominant in the conditioned samples at fields up to $40 \mathrm{kV} / \mathrm{mm}$, but additional hete- rocharge slowly accumulated near the cathode at higher fields, and (c) the space charge densities in the conditioned samples tended to saturate after the voltage had been applied for about 3 months. This additional heterocharge was attributed to electron extraction from low molecular weight $P E$ (presumably at the anode) and drift of the resulting positive ions towards the cathode. Finally, Fleming et al. [7] reported that holding $2 \mathrm{~mm}$ thick planar XLPE samples in short circuit for $48 \mathrm{~h}$ at rotary pump pressure did not prevent space charge accumulation when $\mathrm{dc}$ fields $\sim 20 \mathrm{kV} / \mathrm{mm}$ were applied subsequently. The space charge profile was sensitive to variations in the conditioning time and pressure, and the rate of cooling to room temperature.

Clearly much work remains to be done in order to obtain a more detailed understanding of the influence of the electrodes and heating/ pumping conditioning procedures on space charge formation in XLPE. In this paper we compare space charge profiles for a range of XLPE pla- 

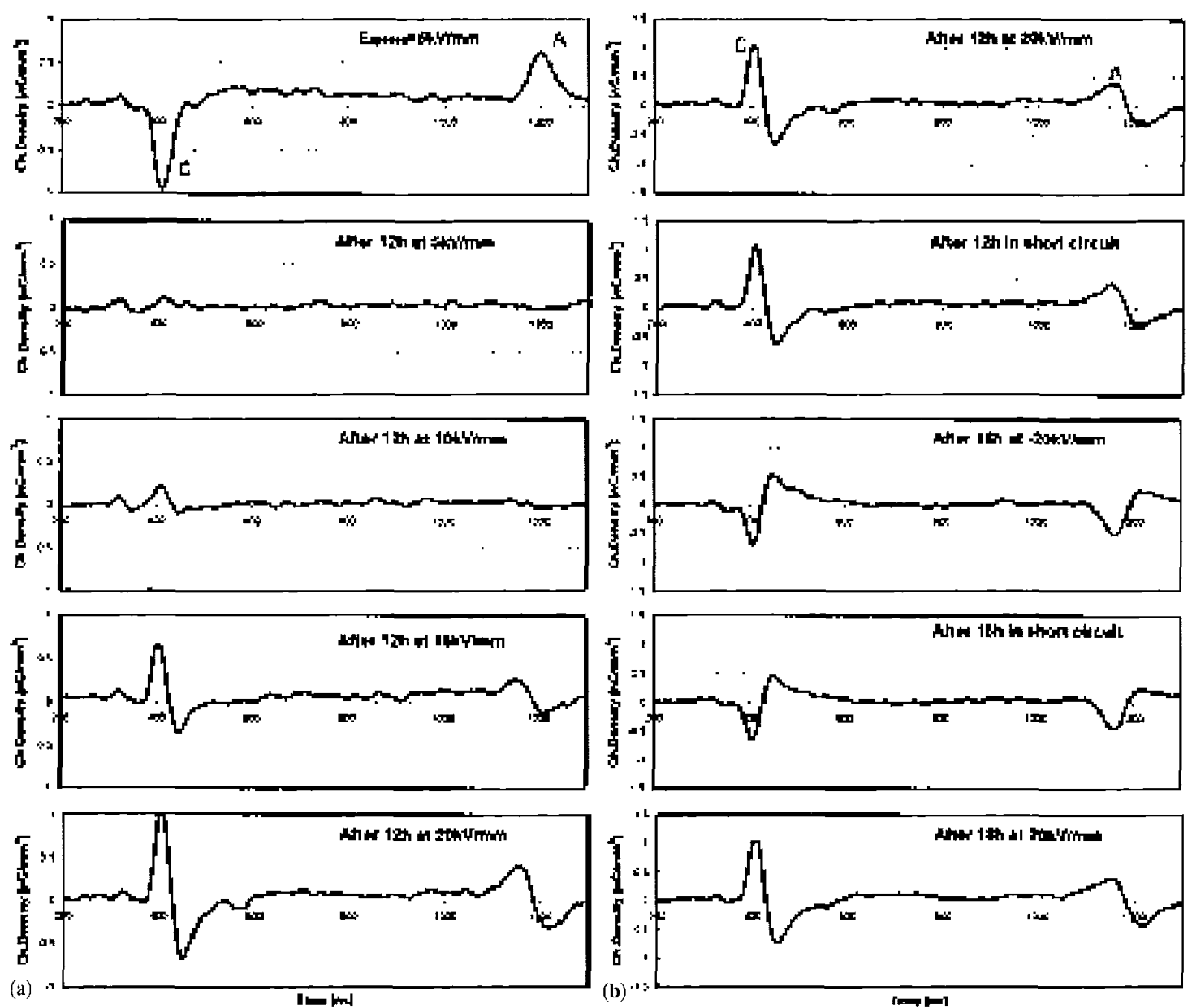

Figure 2. Space charge profiles for a sample with type A semicon electrodes which had undergone HP conditioning. Other conditions as stated for Figure 1.

nar samples under de stress. Three different types of semicon electrodes were investigated, as well as vacuum-evaporated gold. The samples were also subjected to three different heating/pumping conditioning procedures.

\section{EXPERIMENTAL}

\subsection{SAMPLES}

The low density PE (Union Carbide 4201 S) from which the planar samples were fabricated had a melt-flow index of $\sim 2.0$, and contained $\sim 0.2 \%$ wt antioxidant. Crosslinking was achieved using $1.8 \%$ dicumylperoxide. The mixture was maintained at $160^{\circ} \mathrm{C}$ and $2 \times 10^{6} \mathrm{~Pa}$ for $30 \mathrm{~min}$, and then cooled to room temperature over a period of $30 \mathrm{~min}$ by passing water through the press plates. The sample diameter was $145 \mathrm{~mm}$, and the thickness outside the central electrode area was in the range 1.90 to $1.95 \mathrm{~mm}$.

The semicon electrodes, $30 \mathrm{~mm}$ in diameter and $\approx 100 \mu \mathrm{m}$ thick, were attached to the samples by pressing at $160^{\circ} \mathrm{C}$ and $2 \times 10^{6} \mathrm{~Pa}$ for $30 \mathrm{~min}$. This pressing reduced the thickness of the XLPE between the electrodes to $\approx 1.6 \mathrm{~mm}$. The three different types of semicon compound investigated were as follows:
1. Type A, a compound of ethylene-vinyl-acetate and ethylene-acrylicacetate, containing $30 \%$ wt of an acetylene black.

2. Type B, an ethylene-vinyl-acetate based material, containing $32 \%$ wt carbon black, and probably some low molecular weight $\mathrm{PE}$.

3. Type $\mathrm{C}$, an acrylate material containing $36 \%$ wt carbon black.

4. Gold electrodes (Type D) $30 \mathrm{~mm}$ in diameter were vacuum-evaporated on some of the samples (of the same PE and cross-linked as described above). The gold electrode thickness was $\sim 100 \mathrm{~nm}$.

\subsection{CONDITIONING PROCEDURES}

The effects of three different conditioning procedures were investigated, as follows.

Holding at $80^{\circ} \mathrm{C}$ and a pressure of $\sim 10 \mathrm{~Pa}$ (rotary pump pressure) for 4 days, and cooling to room temperature over a period of $\sim 4 \mathrm{~h}$, still at rotary pump pressure (designated heating and pumping (HP)). Holding at $80^{\circ} \mathrm{C}$ and atmospheric pressure for 4 days, and cooling to room temperature over a period of $\sim 4 h$ (designated heating $(H)$ ). Holding at room temperature and a pressure of $\sim 10 \mathrm{~Pa}$ for 7 days (designated pumping (P)).

Some samples were used in their as-received condition (designated unconditioned (U)). 

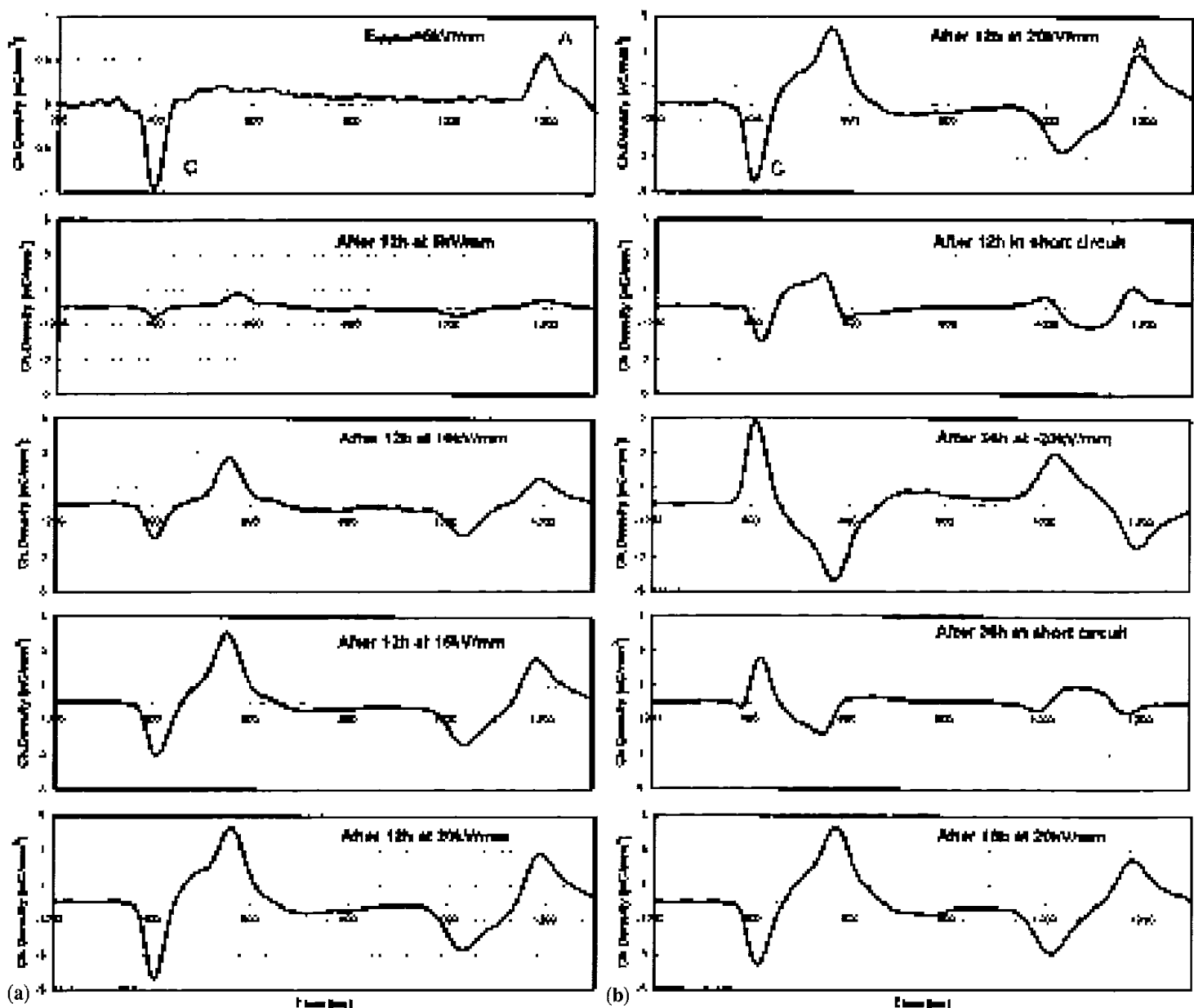

Figure 3. Space charge profiles for a sample with type A semicon electrodes which had undergone P conditioning. Other conditions as stated for Figure 1.

\subsection{SPACE CHARGE MEASUREMENT}

The pulsed electroacoustic (PEA) technique was used to measure the space charge profile [8]. The voltage pulse amplitude and duration were $2 \mathrm{kV}$ and $20 \mathrm{~ns}$ respectively, and the pressure waves were detected using a $28 \mu \mathrm{m}$ thick polyvinylidenefluoride (PVDF) transducer. Each of the space charge profiles shown below was obtained by averaging over 100 voltage pulses. Space charge density calibration was achieved by determining the proportionality constant between the measured signal and the space charge density on the electrode nearer the piezoelectric detector, at low dc field strengths in the range 1 to $5 \mathrm{kV} / \mathrm{mm}$.

\section{RESULTS AND DISCUSSION}

\subsection{SEMICON ELECTRODES}

Figure 1(a) shows five space charge traces for an unconditioned sample with type A semicon electrodes. The top trace was recorded with a field of $+5 \mathrm{kV} / \mathrm{mm}$ applied across the sample, and is presented in order to define the position of the semicon electrodes $(A=$ anode, $C=$ cathode). The field strength is positive in the sense that the HV electrode was at a positive potential relative to the grounded electrode. The latter was in contact with the piezoelectric detector, and the signal associated with it appears near the left-hand edge of each trace. It should be noted that, even at this low field strength, when charge injection from the electrodes and ionization of impurities in the bulk would not be expected, charge appears to have accumulated close to the electrodes. The dispersion of the pressure pulses in transit across the sample is clear from a comparison of the two electrode signals.

The other four traces of Figure 1(a) were recorded after dc fields of various strengths had been applied for $12 \mathrm{~h}$. They were recorded with the sample grounded, and therefore show the space charge in the sample bulk and the charge induced on the electrodes by the bulk charge. The space charge profile changed little during the two hours before each trace was recorded. Heterocharge developed close to the electrodes, saturating at an applied field strength between 10 and $15 \mathrm{kV} / \mathrm{mm}$. The heterocharge peaks are preceded by a point of inflection at 5 and $10 \mathrm{kV} / \mathrm{mm}$, a plateau at $15 \mathrm{kV} / \mathrm{mm}$ and a well-defined minimum at $20 \mathrm{kV} / \mathrm{mm}$. There is a suggestion of similar features close to the anode.

The top trace of Figure 1(b) is identical to the bottom trace of Figure 1(a), and is presented for ease of comparison with the other four traces of Figure 1(b). They were all recorded with the sample grounded. They show the effects of grounding the sample for extended periods af- 

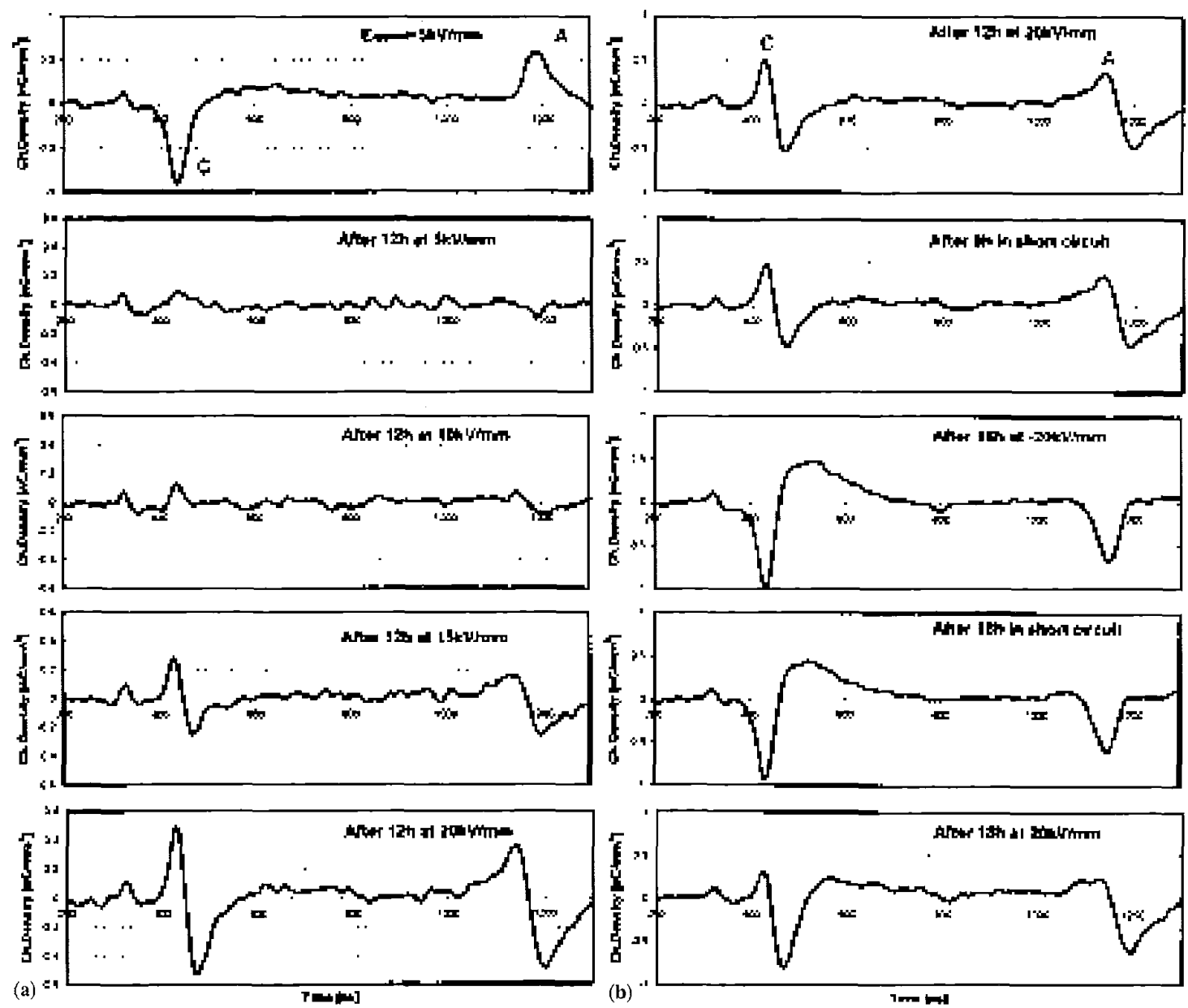

Figure 4. Space charge profiles for a sample with type A semicon electrodes which had undergone H conditioning. Other conditions as stated for Figure 1.

ter poling, and reversing the polarity of the applied field. The accumulated space charge largely disappeared when the sample was grounded for $12 \mathrm{~h}$, and reversal of the field polarity produced the 'mirror image effect' [9], i.e. an almost perfect reflection of the profile in the horizontal axis, except for the charge induced on the electrode surface. The latter is sensitive to the fine details of the charge distribution in the bulk, and it could be that the charge profile, at distances from the electrode shorter than the spatial resolution of the measurements, is polarity dependent. A reversal of polarity could then alter the induced surface charge more than would be expected from a comparison of the macroscopic bulk charge profiles. A second reversal of the applied polarity (bottom trace) produced a profile almost identical to that in the top trace.

The conditions under which the mirror image effect would be expected have been discussed elsewhere [5]. Suffice to say here that the preferred model involves a spatially non-uniform distribution of permanent dipoles in the sample bulk, generating a bound (or polarization) bulk charge density $-\nabla \cdot P$, where $P$ is the volume polarization, and a bound surface charge density $P \cdot n$, where $n$ is the outward drawn normal to the surface. Reversal of the applied field polarity reverses the polarization $P$, which in turn reverses the sign of the bound space charge but does not alter its density. In this way the mirror image effect occurs in the bulk. The spatially non-uniform distribution of permanent dipoles in the sample bulk is thought to originate in residues or byproducts of the crosslinking process, e.g. acetophenone or cumyl alcohol, the non-uniformity resulting from diffusion of these molecules towards the edges of the sample during fabrication. The bound charge density on the surface of the dielectric will induce an equal and opposite charge on the adjacent electrode, but these two components will not be resolved in the present measurements.

The preferred model also involves the injection/extraction of electronic charge across the semicon/XLPE interface by a tunneling mechanism, followed by electron/hole trapping in the bulk close to the interface. This process generates homocharge, and the manner in which it also can lead to the mirror image effect in the bulk, immediately adjacent to the electrodes, has been described in detail elsewhere [9].

We suggest that the Figure $1(a)$ and (b) profiles are generated by the two mechanisms just described, operating simultaneously. Specifically, the two peaks in the bulk close to the cathode result from a combination of injected homocharge, close to the cathode, and bound dipolar heterocharge extending to larger distances from the cathode. The heterocharge dominates close to the electrode, generating an increasing positive signal (for positive applied voltage); over a short distance the 


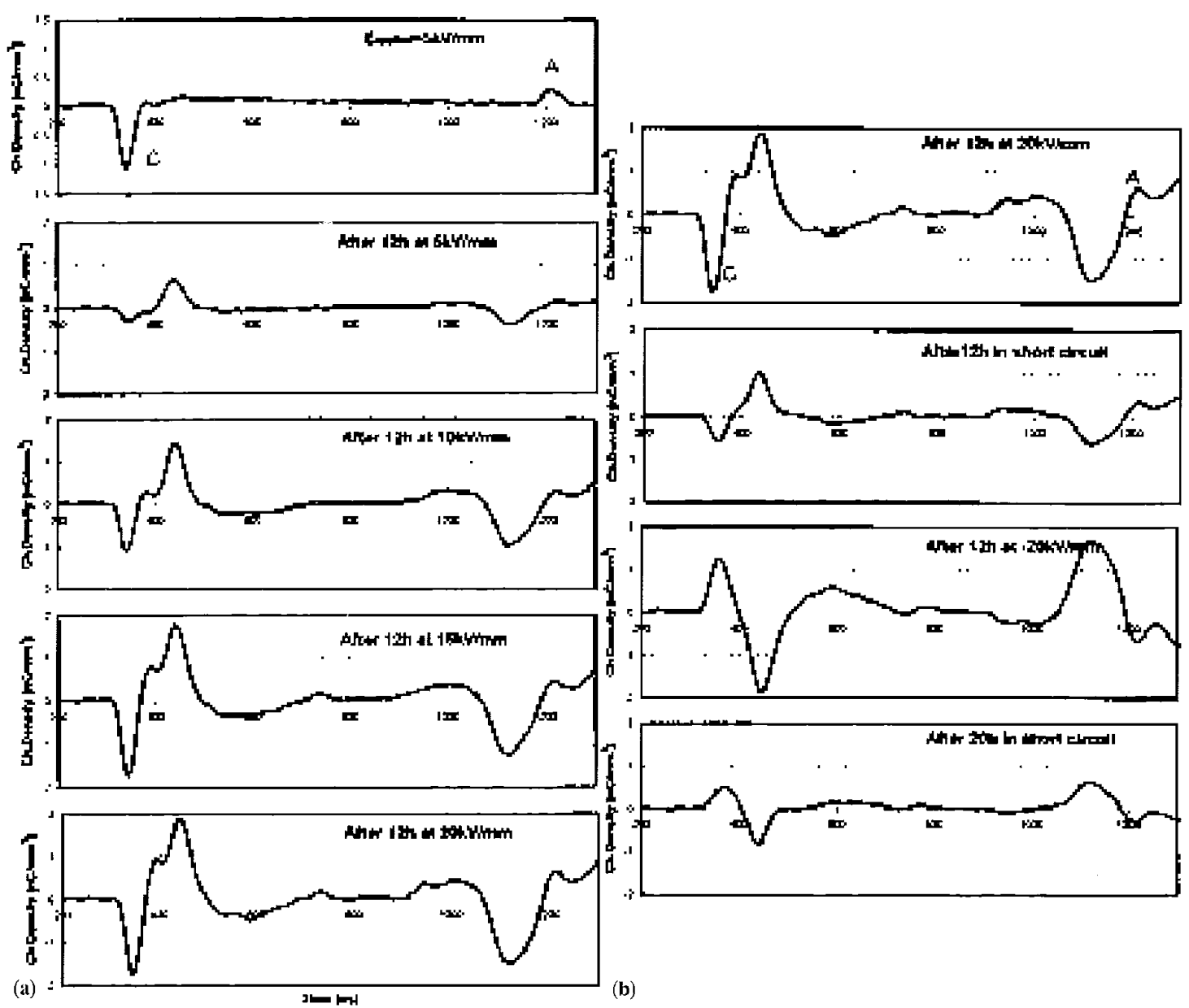

Figure 5. Space charge profiles for an unconditioned sample with vacuum-evaporated gold electrodes. Other conditions as stated for Figure 1.

homocharge density then increases more rapidly, the heterocharge + homocharge sum passing through a point of inflection, a plateau or a welldefined minimum, depending on the rate of increase of the homocharge. The homocharge density then decreases, and the heterocharge passes through a maximum, generating the second positive peak. Finally the heterocharge density falls to zero around the center point of the sample, implying a constant polarization.

The appearance of dipolar heterocharge (rather than dipolar homocharge) implies that the magnitude of the polarization increases with distance from the electrode. The heterocharge peak density doubles between applied field strengths of 5 and $10 \mathrm{kV} / \mathrm{mm}$, as would be expected from the Langevin function describing the field/temperature dependence of the equilibrium orientation of permanent dipoles [10], but then saturates between 10 and $15 \mathrm{kV} / \mathrm{mm}$. The associated inhomogeneous polarization $P$ is proportional to the product of the local dipole concentration and the local field strength. We suggest that the local field strength is reduced by the neighboring injected homocharge, the density of which is still increasing at $20 \mathrm{kV} / \mathrm{mm}$. Presumably the heterocharge peak density would decrease at higher applied fields.

Figures 2(a) and (b) show the corresponding data for a sample with type A semicon electrodes which had been subjected to HP condition- ing. Homocharge appeared immediately adjacent to the electrodes, with no indication of heterocharge. This contrasts strongly with the Figure 1 data. It is difficult to estimate accurately the density of the homocharge implied by Figure 1, but approximate extrapolation indicates that it is roughly double that in Figure 2. The charge profiles changed very little when the sample was grounded for at least $12 \mathrm{~h}$, again contrasting with Figure 1, but the mirror image effect is present, though not to the same accuracy. Considering the differences between Figures 1 and 2, we suggest that the permanent dipoles generating the bound heterocharge are removed by the HP conditioning, but the injection/extraction of electrons across the electrode/XLPE interfaces is little affected.

Figure 3(a) and (b) show data for a sample with type A semicon electrodes which had been subjected to P conditioning. The profiles are similar in shape to those in Figure 1, and the mirror image effect is also clearly visible in Figure 3(b). The profiles differ from those in Figure 1 in that (a) the saturation heterocharge density is $\sim 2 \times$ as large, and (b) the point of inflection associated with homocharge injection does not develop into a plateau and a minimum with increasing applied field strength.

Two other samples which had undergone P conditioning were in- 

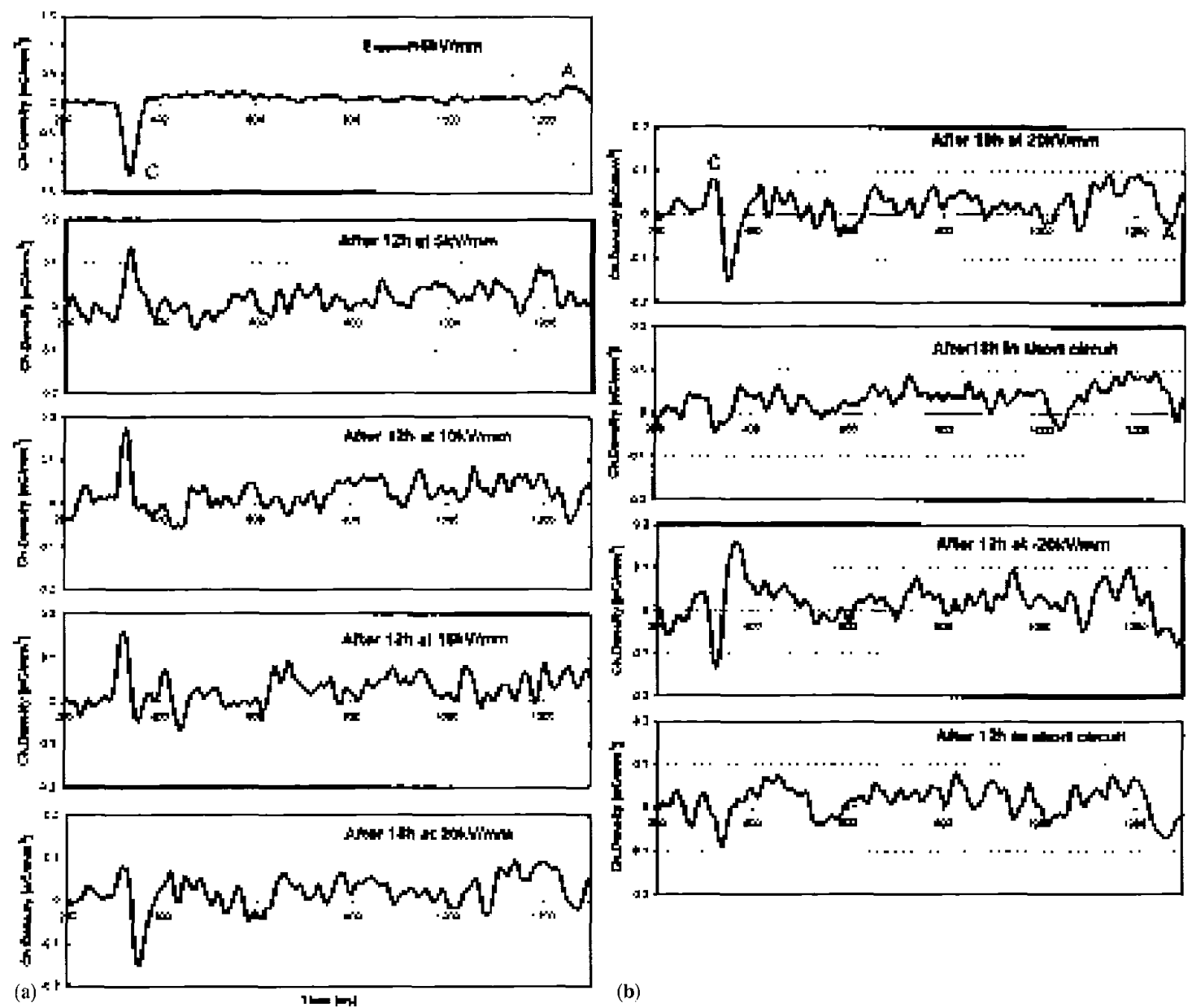

Figure 6. Space charge profiles for a sample with vacuum-evaporated gold electrodes which had undergone HP conditioning, Other conditions as stated for Figure 1.

vestigated, one with type $B$ semicon electrodes and one with gold electrodes. They developed peak heterocharge densities of 3.0 and $4.8 \mathrm{nC} / \mathrm{mm}^{3}$ respectively, after a field of $20 \mathrm{kV} / \mathrm{mm}$ had been applied for $\sim 3 \mathrm{~h}$. The traces for four unconditioned samples (type A, B and $\mathrm{C}$ semicon and gold electrodes) showed maximum heterocharge densities between 1.7 and $2.1 \mathrm{nC} / \mathrm{mm}^{3}$, again after a field of $20 \mathrm{kV} / \mathrm{mm}$ had been applied for $\sim 3 \mathrm{~h}$. It therefore seems likely that $\mathrm{P}$ conditioning produces a significant increase in dipolar bound heterocharge density. The associated mechanism is unclear. It may be that $P$ conditioning removes some of the permanent dipole molecules from the sample volume close to the electrodes, thereby generating a larger dipole concentration gradient and so a larger bound space charge density. Presumably the injection of electronic homocharge occurred in the samples which did not show a point of inflection, but was 'swamped' by the larger heterocharge density.

Figures 4(a) and (b) show data for a sample with type A semicon electrodes which had been subjected to $\mathrm{H}$ conditioning. The homocharge densities are similar to those in Figure 2, and once again the profiles changed little when the sample was short-circuited for $\sim 3 \mathrm{~h}$. A reversal of space charge polarity adjacent to the left-hand-side electrode is evident on reversing the applied field polarity, and is also strongly suggested by the change in the polarity of the charge induced on the right-hand-side electrode. However, the profiles adjacent to the lefthand-side electrode differ considerably on field reversal, and the original profile is not recovered after a second reversal.

\subsection{GOLD ELECTRODES}

The profiles in Figure 5 are for an unconditioned sample with gold electrodes. The heterocharge densities are very similar to those shown in Figure 1, and the mirror image effect, in relation to heterocharge densities and profiles, is seen clearly. The minimum due to homocharge injection, occurring at $t \approx 400$ ns in the top trace of Figure $5(\mathrm{~b})$, did not appear when the applied field polarity was reversed (see third trace), suggesting that electron transfer from gold to these XLPE samples occurs more readily than in the opposite direction.

Figure 6 shows data for a sample with gold electrodes which had undergone HP conditioning. The charge density in the bulk is very close to zero, except following application of $20 \mathrm{kV} / \mathrm{mm}$, at which field strength there is probably some injected homocharge immediately adjacent to the target electrode (bottom trace of Figure 6(a)). Clearly HP 

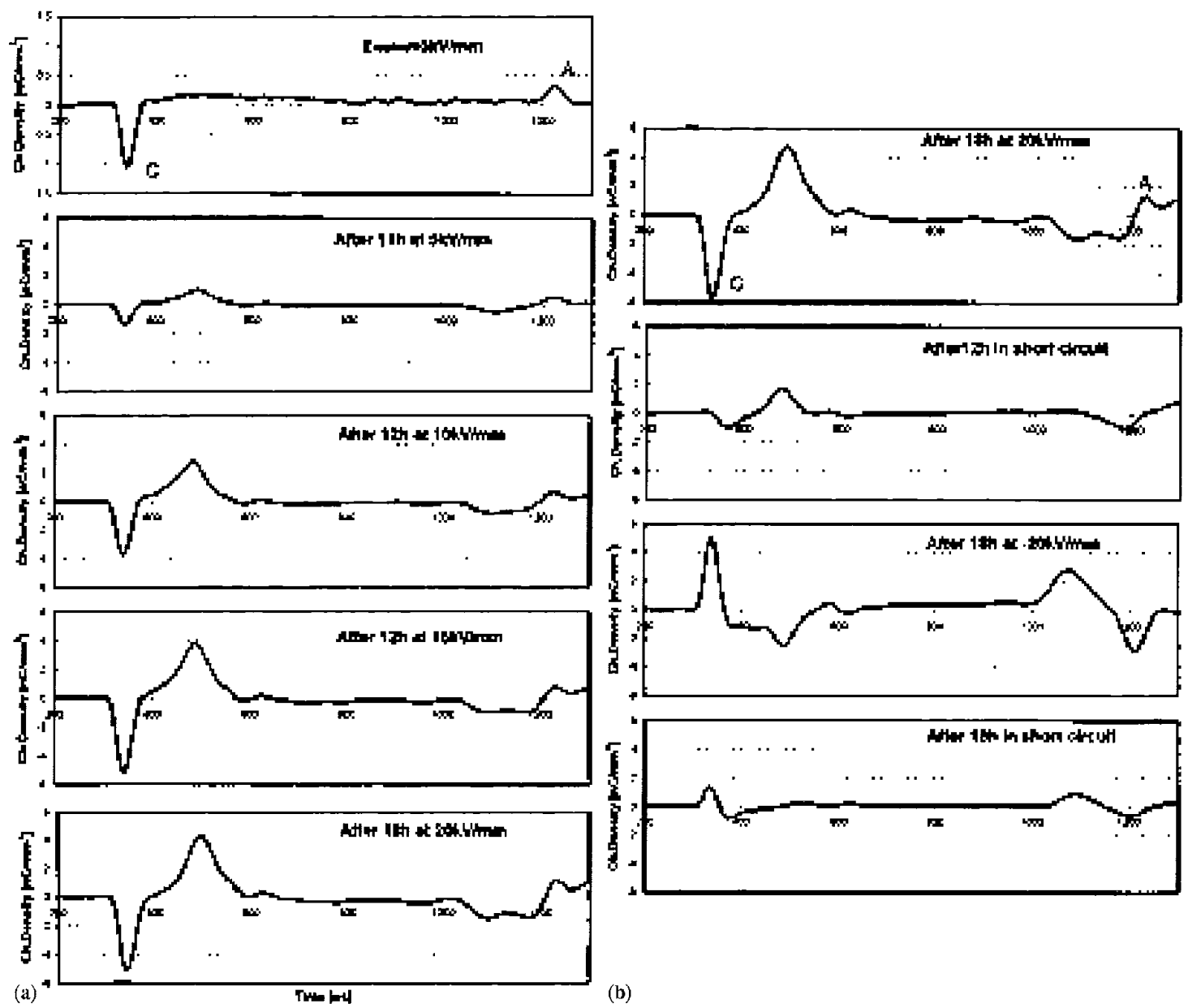

Figure 7. Space charge profiles for a sample with vacuum-evaporated gold electrodes which had undergone P conditioning. Other conditions as stated for Figure 1.

conditioning lowers the rate of injection. The first and third traces of Figure $6(\mathrm{~b})$ near the left-hand electrode are consistent with the mirror image effect.

A sample with gold electrodes subjected to P conditioning (Figure 7) developed, under a positive applied field, heterocharge densities very similar to those in the type A semicon electrode sample subjected to $P$ conditioning (Figure 3). However at $20 \mathrm{kV} / \mathrm{mm}$ it showed homocharge injection (plateau or shallow minimum) only adjacent to the anode, for both field polarities. The heterocharge peak in the $-20 \mathrm{kV} / \mathrm{mm}$ profile of Figure $7(b)$ has only about half the amplitude of the corresponding peak in the top profile, and is preceded by a plateau. Presumably an increase in the density or spatial extent of the injected homocharge reduced the local field strength, and hence the density of the bound heterocharge due to dipole orientation. Since homocharge developed adjacent to the anode, but not adjacent to the cathode, it seems that $P$ conditioning makes electron transfer from XLPE to gold easier than in the opposite direction. This is the opposite of the situation in unconditioned samples with gold electrodes.

Finally, Figure 8 shows profiles for a sample with gold electrodes which had undergone $\mathrm{H}$ conditioning. As expected from Figure 6, the charge densities in the bulk are very small. In Figure 8(a) there is a suggestion of increasing electron injection at the grounded electrode (cathode) with increasing field. In Figure 8(b) the sign of the induced charge on the grounded electrode reverses when the polarity of the applied voltage is reversed, suggesting that the space charge adjacent to the grounded electrode also changes sign.

\subsection{SELF-TRAPPED AND DIPOLE-TRAPPED MONOPOLAR HETEROCHARGE}

The accumulation of monopolar heterocharge in the present samples is considered unlikely for the reasons discussed in [5]. The main evidence against it is the mirror image effect. Why should equal densities of charge of the opposite polarity be trapped in the same locations when the field is reversed?

Self-trapping of charge, resulting from localized polarization surrounding the charge $[11,12]$, is of course a possibility, and would be largely independent of the charge polarity. The effective trap depth can be several $\mathrm{eV}$. This process requires the insulator to contain a polarizable impurity, e.g. water, but the minimum concentration can be quite small, 

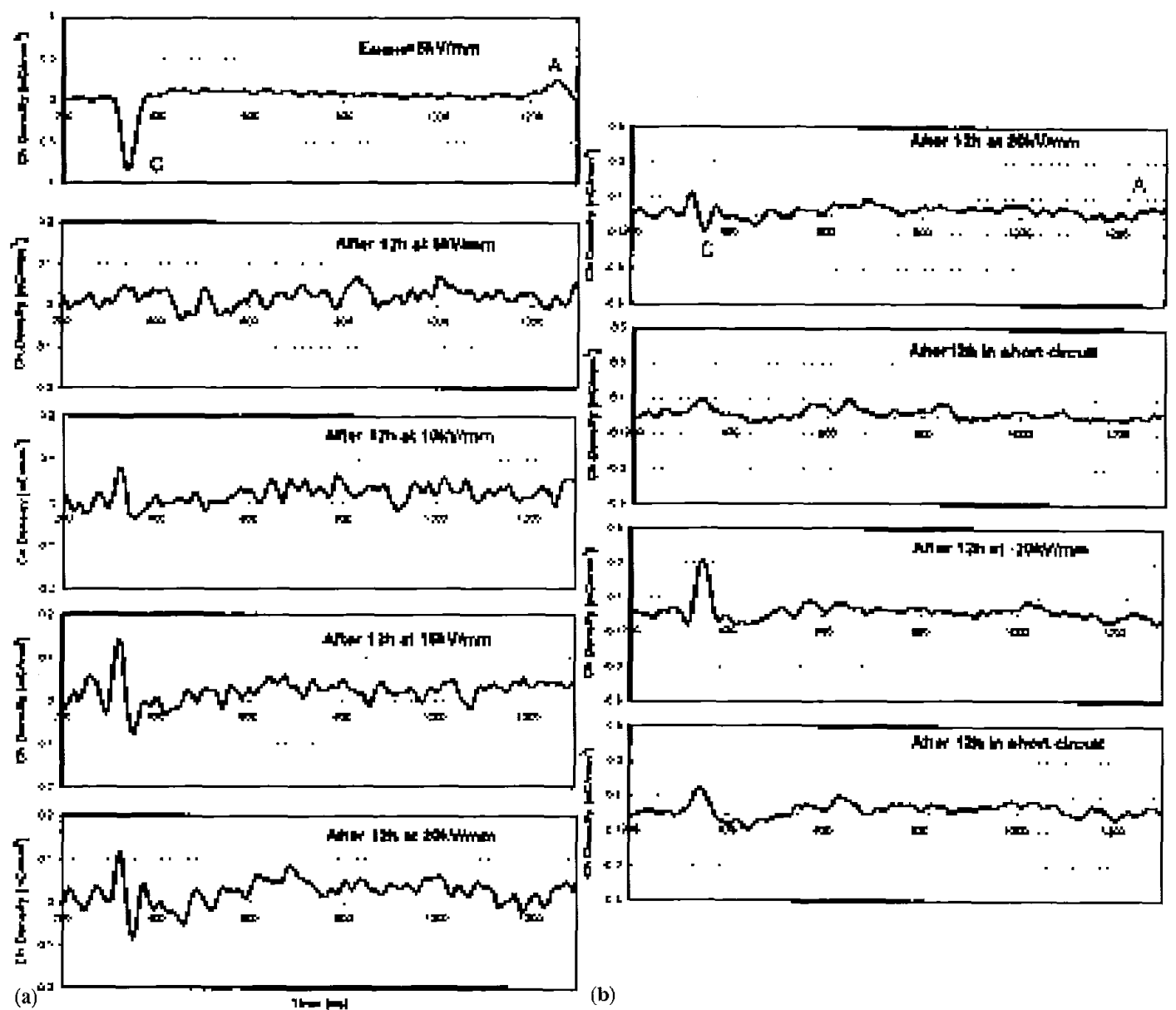

Figure 8. Space charge profiles for a sample with vacuum-evaporated gold electrodes which had undergone $\mathrm{H}$ conditioning. Other conditions as stated for Figure 1.

since the impurity need be present only around the trapping sites. In addition, heating or pumping the sample could easily affect the impurity concentration, at least in the short term, and thus the trapped charge concentration. However, two problems remain in attempting to explain the mirror image effect along these lines. Firstly, why should the self-trapped charge carriers be of the opposite sign when the field is reversed? Secondly, when the sample is short-circuited, monopolar charge would be expected to drift towards the electrodes under its selffield, and be discharged or neutralized there. The present data suggest a slow collapse of the charge in situ.

The trapping of charge by dipoles also is considered unlikely [5], since permanent dipoles with moments $>3.7 \mathrm{D}$ would be required in $\mathrm{XLPE}$, and it is unclear why the trapped charges should be of opposite sign for opposite field polarities.

\subsection{PERMANENT DIPOLE CONCENTRATIONS}

Our preferred mechanism for heterocharge accumulation is the generation of a spatially inhomogeneous polarization due to the orientation of impurity permanent dipoles in the applied field. Such a mechanism is obviously consistent with the mirror image effect, since the polarization vector will reverse direction but maintain the same magnitude when the applied field is reversed. However, it is essential to check whether the implied dipole concentrations are consistent with the expected impurity levels in XLPE. Gauss' law may be written in the form

$$
\varepsilon_{o} \nabla \cdot E=\rho_{f}+\rho_{b}
$$

where $E$ is the electric field, $\rho_{f}$ the free charge density and $\rho_{b}$ is the bound charge density [13]. Assuming negligible free charge density at low applied fields, say $5 \mathrm{kV} / \mathrm{mm}$, then we have

$$
\varepsilon_{o} \nabla \cdot E=-\nabla \cdot P=-\nabla \cdot\left[\chi \varepsilon_{o} E+\frac{N p^{2}}{3 k T} E\right]
$$

where $P$ is the total polarization in the insulation. The latter consists of $\chi \varepsilon_{0} E$, which is the molecular polarization of the $\chi \mathrm{LPE}$, and $\left(N p^{2} / 3 k T\right) E$, which is the polarization due to orientation of the impurity permanent dipoles. $\chi$ is the electric susceptibility of the XLPE, $N$ the dipole density, $p$ the individual dipole moment, and the other symbols have their usual meanings. Since the problem is 1-dimensional, and $\varepsilon_{o} d E(x) / d x=\rho_{b}$ from Equation (1)

$$
\rho_{b}(x)\left[1+\chi+\frac{N(x) p^{2}}{3 \varepsilon_{0} k T}\right]=-\frac{d N(x)}{d x}\left[\frac{p^{2} E(x)}{3 k T}\right]
$$


Note the assumption that the dipolar impurity concentration is sufficiently small that $\varepsilon_{r}$ may be taken as a constant $(\approx 2.3)$ throughout the XLPE volume.

Ideally the equilibrium space-charge density profile would be obtained with the field applied, $\rho_{b}(x)$ and $E(x)$ calculated from the profile, and $N(x)$ then determined piece-wise from Equation (3) using an assumed $N(0)$ value. Unfortunately the equilibrium space charge profiles were obtained in this work only with the sample short-circuited. However, we may reasonably assume that the molecular polarization will largely disappear immediately after the sample is short-circuited, since the electric field strength within a sample rarely differed from the applied field by $>10 \%$ of the latter. This assumption implies setting $\chi=0$ in Equation (3). Given the slow decay of the space charge profiles observed in the present samples, we may also reasonably assume that the dipolar polarization will not relax significantly in the short time ( $<2 \mathrm{~min}$ ) which elapsed between short-circuiting the sample and measuring the space charge profile. Equation (3) then becomes

$$
\rho_{b}(x)\left[1+\frac{N(x) p^{2}}{3 \varepsilon_{o} k T}\right]=-\frac{d N(x)}{d x}\left[\frac{p^{2} E(x)}{3 k T}\right]
$$

Equation (4) is the same as Equation (3) in [5], except that the latter did not contain the $N(x) p^{2} /\left(3 \varepsilon_{o} k T\right)$ term on the left-hand side. Figure 9 shows the variation in $N(x)$ across the sample obtained by applying Equation (4) to the space charge profile shown in Figure 1(a) (after $12 \mathrm{~h}$ at $5 \mathrm{kV} / \mathrm{mm}$ ), for various assumed $N(0)$ values. $E(x)$ was set equal to (short circuit fields $+5 \mathrm{kV} / \mathrm{mm}$ ), and $p$ was taken as $3 \mathrm{D}$, since acetophenone is one of the more abundant dipolar crosslinking products in XLPE [14] and its dipole moment is $\approx 2.86 \mathrm{D}$ [15]. XLPE considered simply as a homogeneous amorphous assembly of $\mathrm{CH}_{2}$ groups, with a density of $900 \mathrm{~kg} / \mathrm{m}^{3}$, will contain $\approx 4 \times 10^{28}$ such groups per $\mathrm{m}^{3}$. The concentration of permanent dipole molecules at the grounded surface of the sample $(x=0)$ was set at zero, $1 \%$ and $2 \%$ of the $\mathrm{CH}_{2}$ group concentration, and the concentration in the remainder of the sample calculated. As expected, it rises to a maximum about one-third of the way across the sample, and then decreases slowly towards the other surface. If the permanent dipoles are crosslinking products and residues, which diffuse towards the sample surfaces as the sample ages, a distribution symmetrical about the mid-point would be expected. However, corrections for dispersion and attenuation of the pressure pulses in transit through the sample were not applied to the present data, and this probably accounts for the asymmetry of the calculated concentrations.

The fact that an assumed dipole concentration of $1 \%$ at $x=0$ leads to a maximum concentration $<3 \%$ is further support for our suggestion that the heterocharge originates in a polarization gradient. In manufacturing the present samples, $1.8 \%$ wt dicumyl peroxide and $0.2 \%$ wt antioxidant were added to the base polymer. Although the percentage of these additives consumed in the crosslinking process is not known, it is possible that other crosslinking products such as cumyl alcohol and $\alpha$-methyl styrene will add to the inhomogeneous polarization. It is also worth mentioning that a concentration of four permanent dipoles per hundred $-\mathrm{CH}_{2}$ - groups in the amorphous regions was deduced from a study of the piezoelectric effect in low density extruded PE cable insulation, assuming a molecular dipole moment of $3 \mathrm{D}[16]$.

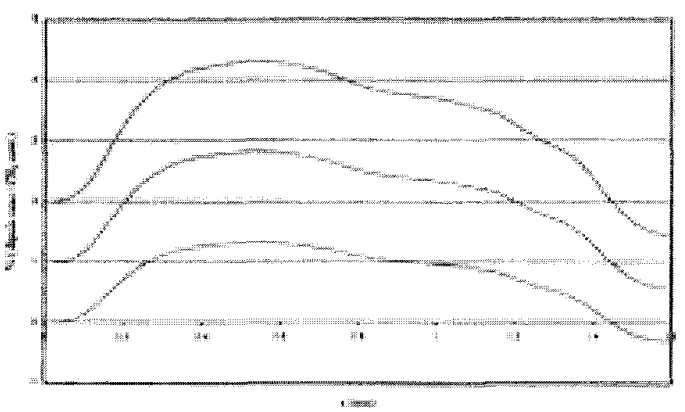

Figure 9. Approximate variation of dipole concentration in an unconditioned sample with type A semicon electrodes, calculated by applying Equation (4) to the profile shown in Figure 1(a), $5 \mathrm{kV} / \mathrm{mm}$. The concentrations are expressed as percentages of the $\mathrm{CH}_{2}$ group concentration in $\mathrm{PE}$, regarded as a homogeneous amorphous $\mathrm{CH}_{2}$ assembly. Three different concentrations were assumed at $x=0$.

Finally, we note that the macroscopic electromagnetic theory of dielectrics predicts an equilibrium space charge density given by $\rho=$ $j \cdot \nabla(\varepsilon / \gamma)$ in a sample carrying a steady dc current of density $j$, and in which the ratio of permittivity $\varepsilon=\varepsilon_{o} \varepsilon_{r}$ to conductivity $\gamma$ is not constant throughout the volume [17]. If the sample is short-circuited this space charge will dissipate at a rate determined by the relaxation time $\varepsilon / \gamma$. The magnitude of the steady state current in polymeric samples equipped with metallic electrodes tends to be independent of the polarity of the applied voltage, and therefore equal and opposite space charge would be expected to accumulate in the sample volume following voltage reversal, provided $\nabla(\varepsilon / \gamma)$ was unchanged. Could the mirror image effect observed in our samples be explained in this way? Any spatial variation in $\varepsilon$ is unlikely to vary with voltage polarity. However, there is no reason to expect that, on reversing the voltage, a positive charge will be trapped at every site where a negative charge was trapped before reversal. Consequently the steady state local electric field $E(x)$ will depend on the voltage polarity, and so will the steady state $\gamma(x)$, since the conduction current density $\gamma(x) E(x)$ is expected to be independent of polarity. Thus both mobile and trapped charge carrier density profiles will be polarity dependent. It seems likely that, on short-circuiting the sample, the mobile carriers which do not undergo recombination will be trapped, and so the space charge profile measured in short circuit will not show the mirror image effect. Nor will space charge generated by ionization of impurities, and therefore consisting mainly of low mobility positive ions.

\section{CONCLUSIONS}

The following conclusions may be drawn from this work:

1. Heterocharge which accumulated in the bulk of unconditioned samples, with semicon or evaporated gold electrodes, was bound charge originating in an inhomogeneous polarization which was itself generated by an inhomogeneous spatial distribution of permanent molecular dipoles.

2. The permanent dipoles were probably acetophenone molecules, one of the by-products of the cross linking reactions. The inhomogeneous spatial distribution, in which the dipole concentration increased in going from the electrodes towards the center of the sample, probably was due to diffusion of the acetophenone in the opposite direction. 
3. The choice of semicon electrode material had little effect on the heterocharge profile in unconditioned samples.

4. Homocharge accumulated in all unconditioned samples close to the electrodes, presumably due to injection/extraciion of electrons across the electrode/ XL,PE interface followed by rapid trapping.

5. Holding the samples at $80^{\circ} \mathrm{C}$ for four days, at rotary pump pressure $(10 \mathrm{~Pa})$ or at atmospheric pressure, suppressed the accumulation of heterocharge, presumably by removing the molecular dipoles. Homocharge accumulation in samples with semicon electrodes was little affected by this conditioning, but was considerably reduced in samples with gold electrodes.

6. Holding the samples at room temperature for seven days at $10 \mathrm{~Pa}$ had little effect on the space charge profiles.

\section{ACKNOWLEDGMENT}

The authors thank NKT Engineering, Denmark for the XLPE samples used in this work, and related technical information. RJF thanks the Tranes Foundation for financial support during his visit to the Department of Electric Power Engineering, Technical University of Denmark, where this work was carried out.

\section{REFERENCES}

[1] R. J. Fleming, "Space charge in polymers, particularly polyethylene", Braz. J. Phys., Vol. 29, pp. 280-294, 1999.

[2] D. Malec, R. Essolbi, H. The-Giam, Bui-Ai and B. Garros, "Space charge and anomalous discharge currents in crosslinked polyethylene", IEEE Trans. on DEI, Vol. 3, pp. $64-69,1996$.

[3] K. S. Suh, C. R. Lee, J. S. Noh, J. Tanaka and D. H. Damon, "Electrical conduction in polyethylene with semiconductive electrodes", IEEE Trans. on DEI, Vol, 1, pp. 224-230, 1994.

[4] X. Wang, D. Tu, T. Muronaka, T. Takada, C. Shinoda and T. Hashizumi, "Space charge in XLPE power cable under de electrical stress and heat treatment", IEEE Trans. on DEI, Vol. 2, pp. 467-474, 1995.

[5] F. N. Lim, R. J. Fleming and R. D. Naybour, "Space charge accumulation in power cable XLPE insulation", IEEE Trans. on DEI, Vol. 6, pp. 273-281, 1999.
[6] B. Sanden, "Space charge accumulation in fresh and degassed XLPE insulation", Space Charge in Solid Dielectrics, ed. J. C. Fothergill and L. A. Dissado, The Dielectrics Society, UK, pp. 225-233, 1998

[7] R. J. Fleming, M. Henriksen and J. T. Holboll, "Charge accumulation in LDPE and XLPE conditioned at $80^{\circ} \mathrm{C}$ under reduced pressure", 1997 Conference on Electrical Insulation and Dielectric Phenomena, pp. 19-22 (IEEE Dielectrics and Electrical Insulation Society, Piscataway, New Jersey, USA).

[8] P. Morshuis and M. Jeroense, "Space charge measurements on impregnated paper: a review of the PEA method and a discussion of results", IEEE EI Mag., Vol. 13 (3), pp. 26-35, 1997.

[9] K. R. Bambery and R. J. Fleming, "Space charge accumulation in two power cable grades of XLPE", IEEE Trans. on DEI, Vol. 5, pp. 103-109, 1998.

[10] A. von Hippel, Dielectric Mnterials and Applications, Artech House, Boston and London, 1995, p. 32. (Originally published as Diclectrics and Waves), A. R. von Hippel, John Wiley and Sons, New York, 1954).

[11] T. J. Lewis, "The micro-physics of charge in dielectrics", Proc, 3rd Int. Conf. on Electric Charge in Solid Insulators, Tours, France), June-July 1998, pp. 16-24 (Societe Francais du Vide, Paris)

[12] G. Blaise and W. J. Sarjeant, "Space charge in dielectrics energy storage and transfer dynamics from atomistic to macroscopic scale", IEEE Trans. on DEI, VoL. 5, pp. 779808,1998 .

[13] P. Lorrain and D. R. Corson, Electromagnetism Principles and Applications, 2nd ed., Ch. 6 (W. H. Freeman \& Co., New York, 1990).

[14] L. A. Dissado and J. C. Fothergill, Electrical Degradation and Breakdown in Polymers, Ch. 9 (Peter Peregrinus Ltd., London, 1992).

[15] J. W. Smith, Electric Dipole Moments, p. 167 (Butterworths Scientific Publications, London, 1955).

[16] R. W. Greaves, E. P. Fowler, A. Goodings and D. R. Lamb, "The direct piezoelectric effect in extruded polyethylene", J. Materials Sci., Vol. 9, pp. 1602-1608, 1974.

[17] I. W. McAllister, G. C. Crichton and A. Pedersen, "Charge accumulation in dc cables: a macroscopic approach", Conference Record of the 1994 IEEE Int. Symp. on Electrical Insulation, Pittsburgh, Pennsylvania, USA, pp. 212-216 (IEEE, Piscataway, New Jersey, USA)

1 Permanent address: Dept. of Physics, Monash University, Clayton, Victoria, Autralia.

This manuscript is based on a paper given at the 10th International Symposium on Electrets, Delphi, Greece, 22-24 September 1999.

Manuscript was received on 14 March 2000, in final form 8 June 2000. 